NIST Technical Note 1648

\title{
Delivering Building Intelligence to First Responders
}

David G. Holmberg

Michelle A. Raymond

Jason Averill

http://dx.doi.org/10.6028/NIST.TN.1648

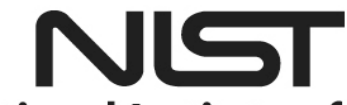

National Institute of Standards and Technology U.S. Department of Commerce 


\title{
Delivering Building Intelligence to First Responders
}

\author{
David G. Holmberg \\ Energy and Environment Division \\ Engineering Laboratory \\ Jason Averill \\ Fire Research Division \\ Engineering Laboratory \\ Michelle A. Raymond \\ Honeywell Laboratories
}

http://dx.doi.org/10.6028/NIST.TN.1648

February 2013

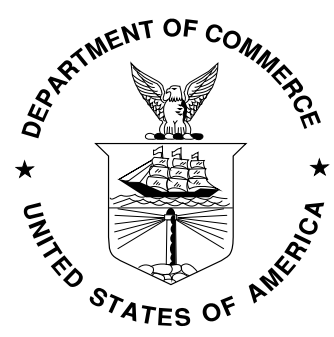

U.S. Department of Commerce Rebecca Blank, Acting Secretary

National Institute of Standards and Technology Patrick D. Gallagher, Under Secretary of Commerce for Standards and Technology and Director 
Certain commercial entities, equipment, or materials may be identified in this document in order to describe an experimental procedure or concept adequately. Such identification is not intended to imply recommendation or endorsement by the National Institute of Standards and Technology, nor is it intended to imply that the entities, materials, or equipment are necessarily the best available for the purpose.

National Institute of Standards and Technology Technical Note 1648 Natl. Inst. Stand. Technol. Tech. Note 1648, 19 pages (February 2013) http://dx.doi.org/10.6028/NIST.TN.1648 CODEN: NTNOEF 


\begin{abstract}
Presently, there is no integrated solution for delivering building systems data to emergency responders. A standard building data delivery solution will integrate the capacity of the building fire safety systems with the capabilities of the fire service, resulting in an improvement in firefighter safety and a reduction in commercial property losses due to fire. Remote access to these data would provide greatly improved situational awareness for emergency responders, reducing response times as well as time to size up and mitigate building fires.

This paper reviews challenges of collecting, processing, and moving sensor data out of building systems, meshing with public safety networks and addressing network architecture and security issues. The paper then presents a summary of the current stateof-the-art, including a proposed data classification system. Finally, this paper presents a roadmap to address remaining challenges in enabling first responder access to critical building incident data.
\end{abstract}

Keywords: alarms; building automation; building information modeling; communications; data classification; emergency response; fire; fire alarm; fire service; networks; standards

\title{
Introduction
}

Fire service response to a commercial or industrial building is typically initiated with a fire sensor signal relayed to an alarm company, followed by a 9-1-1 call. Presently, the information that reaches first responders about the fire incident is minimal. Consequently, standard operating procedure upon arrival at a building site is based on minimal situational awareness, requiring significant time after arrival to conduct an on-scene assessment before beginning suppression action. A goal of the National Institute of Standards and Technology (NIST) Building and Fire Research Laboratory has been to enhance situational awareness of firefighters responding to building emergencies. Situational awareness for the fire service requires knowledge of available assets, operational capabilities, and information about the nature of rapidly evolving hazards. Real-time display can provide critical knowledge to an incident commander about the location, magnitude, and vector of the fire in a geospatial context. Real-time access to building systems data holds the potential to improve fire service safety and response capabilities.

Modern building automation systems have a wealth of sensor data which, when properly collected, analyzed, and communicated, can be made available remotely to responders and public safety networks in secure, interoperable formats. The goal is to enable a future where first responders can arrive at a building already knowing the magnitude and 
location and hazards (including both emerging hazards such as fire and smoke, as well as fixed hazards such as stored chemicals or explosives), quickly perform an on-scene sizeup, and deploy resources appropriate to the risks, enhanced by real-time alerts showing conditions and assets within the context of the building and site. Ultimately, the goal of this research is to improve firefighter safety by reducing the over 83,000 injuries each year in the U.S. and reducing property losses in structures (which totaled $\$ 11.8 \mathrm{~B}$ in 2005) [1].

There are many challenges to delivering real-time building information to first responders. First, there are security concerns when linking a private building control system to a public safety network. Second, there is no framework in place for identifying, formatting, and moving building data to authorized end users. Third, there are multiple stakeholders and stakeholder networks that must interface in order to move alarm data from a building via the Next Generation 9-1-1 network to a public safety answering point (PSAP) and then to dispatch and ultimately to responding units who must then connect to the building for real-time data access. Finally, there are building code and certification (such as Underwriters Laboratories certification) barriers to interactions with life safety systems, and non-interoperable legacy control systems that hinder communications.

This paper presents an organization of the work tasks that must be addressed to move forward on delivery of building information in real-time for emergency response, and focuses on the work done to address building information identification, classification and spatial representation of that information to allow presentation to the emergency responder.

\section{Previous Work}

Over the past two decades, building automation systems (BAS) have become more intelligent, automated, and connected to the outside world; however, the fire service has not derived the full benefits of these innovations. State-of-the-art BAS integrate a variety of building services including heating, ventilation, and air-conditioning (HVAC), lighting control, access control, and fire detection systems. However, that rich information is not currently able to reach emergency responders. Starting in 2004, NIST worked with industry partners and end-users to enable secure real-time communication of building system information to emergency responders by developing an information exchange architecture, data taxonomy, standards, analysis methods, and performance measures.

NIST began addressing building information for first responders in 2004 [2] by working with fire and police representatives to identify types of building information that would be critical for faster and safer building incident response. These categories of building information were subsequently included in the National Electrical Manufacturers 
Association (NEMA) Standards Publication SB 30-2005 Fire Service Annunciator and Interface [3]. NEMA SB30 standardizes the information content and display format of building data and interfaces to real-time information for use by the fire service incident commanders and first responders. The SB 30 standard specifies a consistent layout for display to make it easier for the fire service to interact with fire panels from multiple vendors [4]. Additionally, the NEMA SB30 standard has been included as an adoptable annex (Annex F) within the 2007 edition of the National Fire Alarm Code (NFPA 72) [5].

In 2006 and 2007 [6], NIST expanded the scope of the work beyond standards for display of building information to include standard methods for accessing building information and moving the data over public safety networks to emergency dispatch and to responders en-route [7]. The 2007 proof-of-concept demonstration showed moving building information to responding apparatus en-route was possible, yet many significant challenges remain. To develop a comprehensive approach to these challenges, a stakeholder workshop was held in October 2008 [8]. This paper summarizes workshop results and provides documentation of subsequent work efforts.

\section{Fire Response in 2020}

The following scenario presents the vision for future emergency response with integrated building information presentation. A hypothetical commercial laboratory facility has a small fire occur afterhours. The facility is equipped with a modern building automation system that initiates a sequence of notifications according to a prescribed communications protocol: the BAS receives a fire detection signal from an addressable sensor within the fire alarm system and sends the alarm indicator to a monitoring company via a Standard Access Point (SAP) on the building information services network, which then relays the signal via the Next Generation 9-1-1 (NG911) system to the Public Safety Answering Point (PSAP). This information is automatically forwarded to the computer aided dispatch (CAD) system where a dispatcher views the information on a computer display in the context of geospatial and other key information. Seeing a report of a small fire in a sixth floor lab, the dispatcher sends appropriate units. Based on the building's contact policy at the correct point(s) along this chain, the authorized building personnel on site and building owner are apprised of the current situation.

Two minutes later, a second alarm is activated in the lab facility. The BAS sends a standard alarm signal out with building ID, incident ID, and alarm location code. Because a previous alert has followed this path already, the subsequent information travels nearly instantaneously to the CAD system which links this alarm to the previously received alarm. The dispatcher and first responders can now see how the fire is growing. The dispatcher sends additional units. 
The alarm signals, as they continue to arrive, are communicated verbally to responders over radio communication systems or as a generated audio file via standard data transmissions. Regardless of the audio component distribution method, standard data transmissions are always sent to provide graphical building and geographic information system (GIS) representations through the secure public service network. En-route responders have access to a visual display of the building footprint and surrounding roads. This display draws on both real-time situation alerts forwarded from dispatch as well as stored building floor plan and firefighting equipment location data. The incident commander can see the building footprint and interior plans, location of fire hydrants, stairwells and standpipes, get status updates on the progression of the fire, determine the best locations for vehicle staging and building access, and develop a risk-informed strategy for rescue and suppression operations.

The first truck arrives five minutes after the first fire alarm signal activates, before any smoke is visible outside the building. Once incident command is established, the incident commander (IC) can utilize the floor plan display that shows clearly where the fire is, where the smoke is, an incident alarm history, laboratory hazards (such as pressurized cylinders and flammable or explosive liquids), stairwell access, standpipes, utility shutoff locations, and other needed information. The IC can see where the fire began and how it is spreading, along with smoke conditions. Rather than spending valuable time walking the floors of the building to find the fire, then calling in reinforcements, the IC starts implementing a plan for mitigation even before the additional units arrive. By entering the building at an earlier stage in the fire development, with a clear suppression and rescue plan, firefighters are exposed to less severe interior conditions, property damage is minimized, and opportunities for civilian rescue are maximized.

\section{The building information delivery path}

The above scenario presumes a number of critical standards are in place and in use across a given jurisdiction. These include standards for: network interface to the building; classifying, formatting, and moving data securely across public safety networks; presentation of building information; and other interoperability standards.

Today's data path is very simple, as shown in Figure 1. A simple alarm notification from the fire panel reaches the central station alarm (CSA) monitoring company via a connection that is typically proprietary. A CSA operator then calls 9-1-1 and verbally communicates "fire alarm reported at XYZ address." The call taker enters this basic information into the CAD system and forwards to a dispatcher. A responder typically knows only that an alarm has been reported at an address, until they arrive at the scene, although additional information may be called in. 


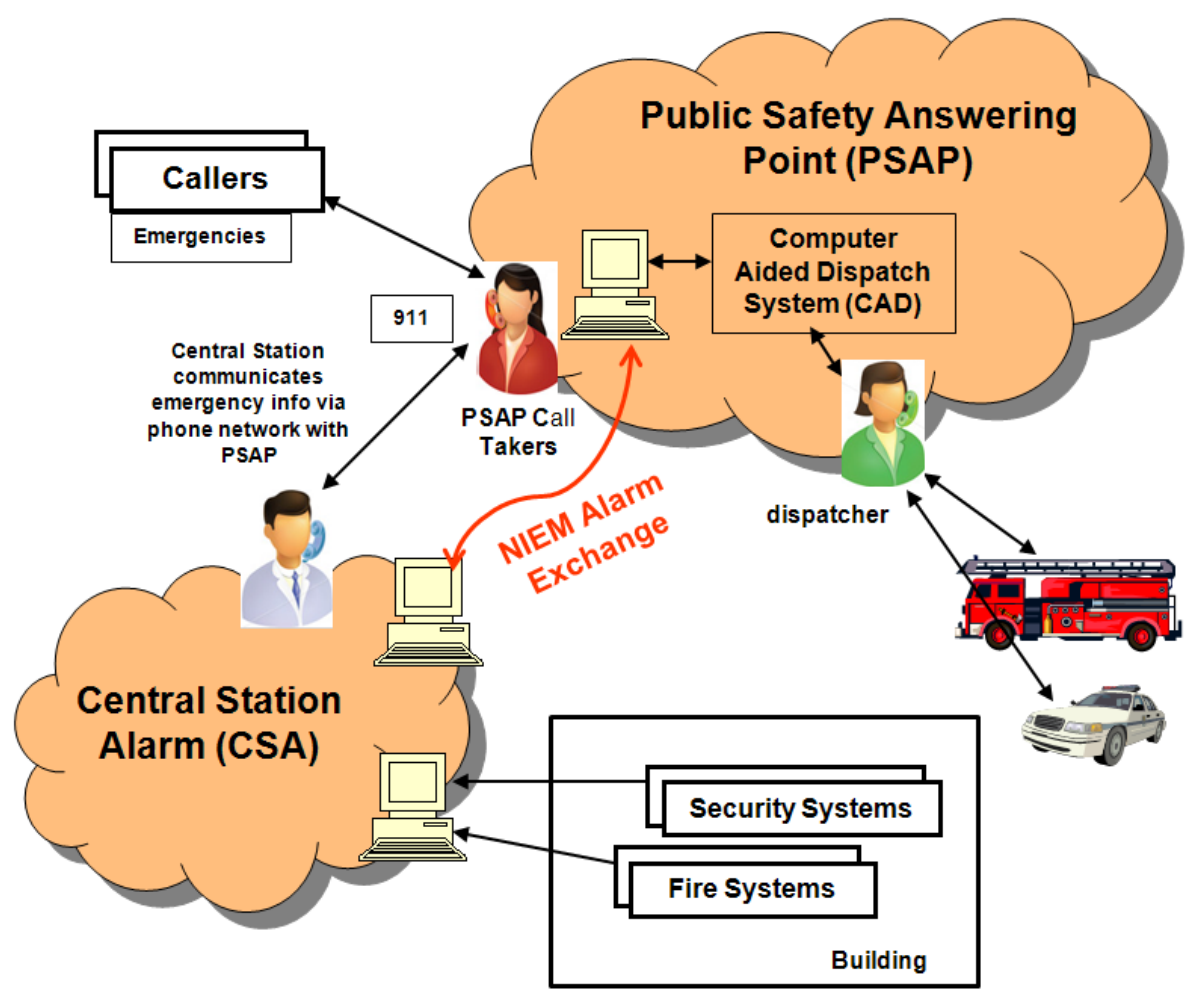

Figure 12009 Building alarm information route

Figure 2 shows the data path that will enable the 2020 scenario. The building has a standard access point (SAP) through which alerts and alarms are distributed to authorized subscribers. The CSA company monitors the alerts and alarm notifications coming from the building SAP and relays alarms via the NG911 network to the PSAP. The PSAP internally routes information to the CAD system which passes alarm information to dispatched responders. Computers in the vehicles receive real-time data updates (from dispatchers as well as directly from the building SAP), and can present the updates in useful ways, both audibly and visually, en-route and on-scene, to speed size-up and mitigation. 


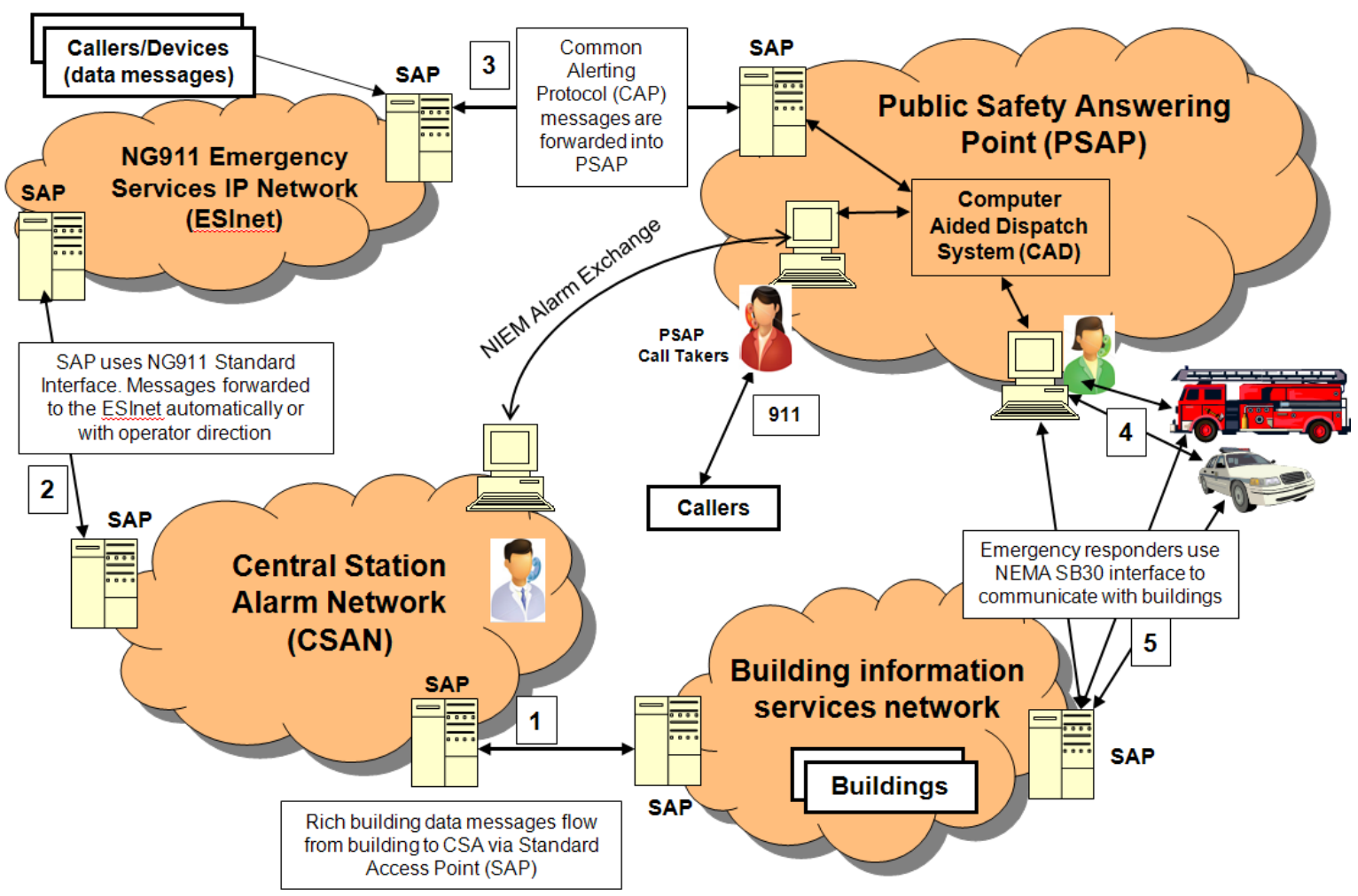

Figure 2 The next generation data network path for NG911, CSA, PSAP and building network integration. The building alert message progresses along the sequence from building to responder as indicated by the numbered boxes from [1] to [5].

Figure 2 also helps one see the many challenges related to network interconnections, security architecture, information sharing protocols, and the collection, classification, transport, and presentation of building systems data. These work items are discussed in the following section.

\section{Work tasks and status}

The NIST October, 2008 stakeholder workshop identified the technical challenges and began the process of identifying work items and forming a strategic approach. As summarized in Table 1, task group efforts since the workshop have organized the major technology component tasks. Examination of these work tasks has shown that each component specification (work progress on individual components) impacts all other components; that is, moving forward on any one task impacts all other tasks. The components in this diagram will be reviewed in order to gauge progress in the work.

Table 1 Technology components and key subcomponents to address for building information delivery to emergency responders

\begin{tabular}{|l|l|}
\hline Message Contents & $\cdot \begin{array}{l}\text { Identification of mission-critical building information for } \\
\text { emergency response } \\
\text { Data classification }\end{array}$ \\
\hline
\end{tabular}




\begin{tabular}{|c|c|}
\hline Message Formats & $\begin{array}{ll}\text { - } & \text { Alert messages } \\
\text { - } & \text { Information exchange package documentation } \\
& \text { Distribution wrappers }\end{array}$ \\
\hline Message Distribution & $\begin{array}{l}\text { - } \quad \text { Process rules adherence } \\
\text { - } \quad \text { Alert recipients } \\
\text { - Data request and response process }\end{array}$ \\
\hline IT Infrastructure & $\begin{array}{l}\text { - } \quad \text { Building information servers } \\
\text { - } \quad \text { Standard access points } \\
\text { - Networks }\end{array}$ \\
\hline $\begin{array}{l}\text { Graphical User } \\
\text { Interface }\end{array}$ & $\begin{array}{l}\text { - } \quad \text { Display layout } \\
\text { - User activity-based interaction } \\
\text { - User role-based interaction }\end{array}$ \\
\hline $\begin{array}{l}\text { Spatial } \\
\text { Representation }\end{array}$ & $\begin{array}{ll} & \text { Floor plan model and information exchange } \\
\text { - } & \text { Space naming conventions } \\
\text { - } & \text { Alert to space association }\end{array}$ \\
\hline User Profiles & $\begin{array}{l}\text { - } \quad \text { User roles } \\
\text { - } \quad \text { Role-based information requirements } \\
\text { - Situation-based user activation and role assignment }\end{array}$ \\
\hline Information Security & $\begin{array}{l}\text { - User access control } \\
\text { - Data transport and storage } \\
\text { - } \quad \text { Process rules adherence }\end{array}$ \\
\hline
\end{tabular}

The overarching challenges of structuring information for situational awareness in emergency response were identified and approaches recommended in previous work [9, 10]. Structuring information challenges cross into all technology components $[11,12,13]$.

\section{Message Contents}

The Message Contents component addresses the identification and classification of building information that is of use to emergency responders, and the grouping of that data to be of use to different end users. As indicated earlier, significant work has already been done at NIST and elsewhere to identify the set of building information that is mission critical for emergency response-including fire, law enforcement, and emergency medical applications [14].

The aforementioned output towards identifying critical building system data has been incorporated in to the NEMA SB30 standard. One of the foundational elements that will allow for standardized public safety access to building system data is a classification system leveraging classification developments in the architecture and engineering domain such as OmniClass ${ }^{\mathrm{TM}}$ [15]. This provides a common vocabulary and organization to the data that allows for standard messages to request specific data elements or sub-sets of the data and which, in turn, allows standardized presentation of the data. In other words, there must be agreed upon data classification to map building data in the building system on one side to NEMA SB30 on the other side. 
Tables 2 and 3 present a proposed data classification hierarchy that shows how building data can be organized to enable standardized groupings. Table 2 presents a data classification of static information; Table 3 presents dynamic (real-time sensor) data. The left-most columns in each table show which of these data elements are required or optional in each of the three NEMA SB30 user interface displays: en-route display, incident commander (IC) display, and fixed fire panel display. The en-route display presents information needed by the responders while on the road: building address and orientation, alarm and sprinkler status, fire conditions, hazards, etc. The incident commander display presents more detailed information on a floor plan display: locations of equipment, hazards, alarms, and fire condition information. The fixed fire panel display remains in the building and has essentially the same information as the remote IC display, but allows building control actions not enabled remotely (e.g., silence alarms).

Table 2 Building Static Data proposed data classification

\begin{tabular}{|c|c|c|c|c|}
\hline \multicolumn{4}{|c|}{$\begin{array}{c}\text { NEMA SB30 } \\
\text { display }\end{array}$} & \multirow[b]{2}{*}{ Static Data Hierarchy } \\
\hline \multirow[t]{2}{*}{ 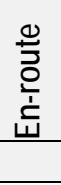 } & 0 & 힐 & 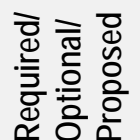 & \\
\hline & & & & Building/Static Data/ \\
\hline \multirow[b]{2}{*}{$\mathrm{X}$} & & & & /Metadata \\
\hline & & $\mathrm{X}$ & Req & $\begin{array}{l}\text { - } \quad \text { Building Identification (ID Number, address) } \\
\text { - } \quad \text { Compass (lat long, north, side labels) }\end{array}$ \\
\hline \multirow[t]{2}{*}{$\mathrm{X}$} & $\mathrm{X}$ & $\mathrm{X}$ & Opt & - Building information (use, age, stories, sprinkler, etc.) \\
\hline & $\mathrm{X}$ & $\mathrm{X}$ & Opt & - Contacts (type, name, number) \\
\hline & & & & /Floor Plans \\
\hline $\mathrm{X}$ & $\mathrm{X}$ & $\mathrm{X}$ & Req & - Walls (location, construction) \\
\hline $\mathrm{X}$ & $\mathrm{X}$ & $\mathrm{X}$ & Req & - Doors (location, construction, normally open/closed/locked) \\
\hline $\mathrm{X}$ & $\mathrm{X}$ & $\mathrm{X}$ & Req & - Window (location) \\
\hline $\mathrm{X}$ & $\mathrm{X}$ & $\mathrm{X}$ & Req & - Stairs (location, roof access) \\
\hline \multirow[t]{4}{*}{$\mathrm{X}$} & $\mathrm{X}$ & $\mathrm{X}$ & Req & - Elevators (location, range of floors) \\
\hline & $\mathrm{X}$ & $\mathrm{X}$ & Req & - Exits (location) \\
\hline & $\mathrm{X}$ & $\mathrm{X}$ & Req & - Floor plan (floor name, spaces, label, type, location, building element) \\
\hline & $\mathrm{X}$ & $\mathrm{X}$ & Req & - Roof plan (access door, roof construction, heavy object, air vent outlet) \\
\hline & & & & /Features/ fire response features \\
\hline $\mathrm{X}$ & $\mathrm{X}$ & $\mathrm{X}$ & Req & - Water access (standpipe, firefighter connection) \\
\hline $\mathrm{X}$ & $\mathrm{X}$ & $\mathrm{X}$ & Req & - Areas of refuge (location) \\
\hline $\mathrm{X}$ & $\mathrm{X}$ & $\mathrm{X}$ & Req & - Firefighter elevators (location) \\
\hline $\mathrm{X}$ & $\mathrm{X}$ & $\mathrm{X}$ & Req & - $\quad$ Firefighter entrances (location) \\
\hline \multirow[t]{6}{*}{$\mathrm{X}$} & $\mathrm{X}$ & $\mathrm{X}$ & Req & - Fire panels (location) \\
\hline & $\mathrm{X}$ & $\mathrm{X}$ & Req & - Utility shutoffs (gas, electric, HVAC, sprinkler locations) \\
\hline & $\mathrm{X}$ & $\mathrm{X}$ & Req & - Fire displays (location) \\
\hline & $\mathrm{X}$ & $\mathrm{X}$ & Req & - Fire phones (location) \\
\hline & $\mathrm{X}$ & $\mathrm{X}$ & Req & - Fire fighter gears (pre-positioned gear, airpack refill station) \\
\hline & $\mathrm{X}$ & $\mathrm{X}$ & Req & - Halon systems (location) \\
\hline
\end{tabular}




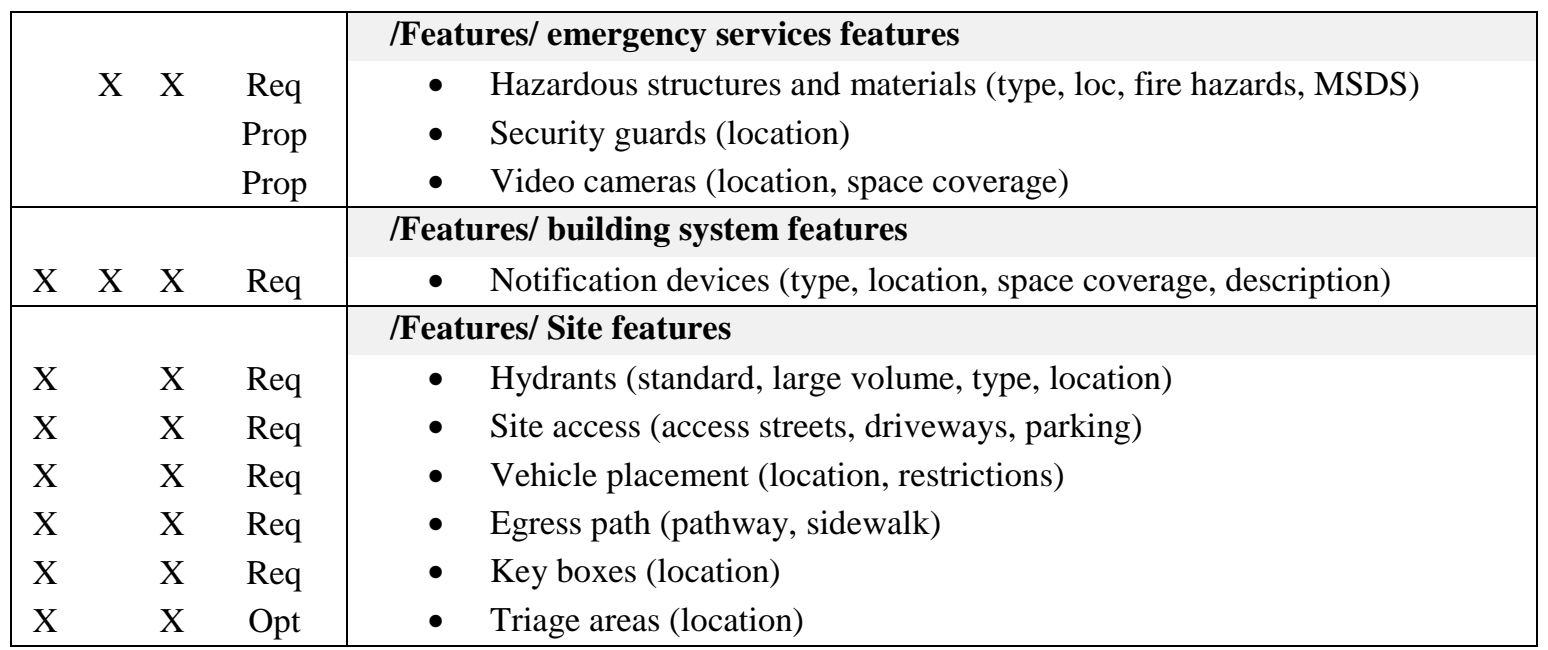

Table 3 Building real-time data proposed data classification

\begin{tabular}{|c|c|c|c|c|}
\hline \multicolumn{4}{|c|}{$\begin{array}{c}\text { NEMA SB30 } \\
\text { display }\end{array}$} & \multirow[b]{2}{*}{ Real-time Data Hierarchy } \\
\hline \multirow[t]{2}{*}{ 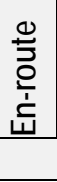 } & $\underline{\underline{u}}$ & $\begin{array}{l}\text { ge } \\
\dot{d} \\
\end{array}$ & 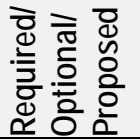 & \\
\hline & & & & Building/Real-time Data/ \\
\hline \multirow{6}{*}{$\begin{array}{l}X \\
X \\
X\end{array}$} & & & & /Building system status \\
\hline & & & & - $\quad$ Fire alarm status \\
\hline & $\mathrm{X}$ & $\mathrm{X}$ & Req & o First alarm (type, location, description, time) \\
\hline & $\mathrm{X}$ & $\mathrm{X}$ & Req & o Most recent alarm (type, location, description, time) \\
\hline & $\mathrm{X}$ & $\mathrm{X}$ & Req & o Alarm list (type, loc, description, time, supervisory/trouble) \\
\hline & & & Prop & o $\quad$ Fire extent (progression of fire alarms) \\
\hline \multirow[t]{3}{*}{$\mathrm{X}$} & $\mathrm{X}$ & $\mathrm{X}$ & Opt & - Sprinkler system status (off, flowing, trouble) \\
\hline & $\mathrm{X}$ & $\mathrm{X}$ & Opt & - Smoke control system status (on/off, pressure sensor location) \\
\hline & $\mathrm{X}$ & $\mathrm{X}$ & Opt & $\begin{array}{l}\text { - Elevator status (operating/disabled, alarms, current floor, direction of } \\
\text { movement, destination floor) }\end{array}$ \\
\hline \multirow[t]{9}{*}{$X$} & $\mathrm{X}$ & $\mathrm{X}$ & Opt & - Utility shutoff status (on/off) \\
\hline & $\mathrm{X}$ & $\mathrm{X}$ & Opt & - Sensor status (type, alert) \\
\hline & $\mathrm{X}$ & $\mathrm{X}$ & Opt & - Video data (camera) \\
\hline & $\mathrm{X}$ & $\mathrm{X}$ & Opt & - Building occupancy (occupant number, location, certainty) \\
\hline & & & Prop & - Security system alarms \\
\hline & & & Prop & - Lighting system status \\
\hline & & & Prop & $\begin{array}{l}\text { /first responder status (location, identifier, qualifications, physiological } \\
\text { condition, remaining time on airpack, alert on man down) }\end{array}$ \\
\hline & & & Prop & $\begin{array}{l}\text { /fire decision support (space heat release rate, visibility, flashover potential, } \\
\text { collapse warning) }\end{array}$ \\
\hline & & & Prop & $\begin{array}{l}\text { /control functions (silence notification appliance, ack fire event, reset fire } \\
\text { system alarm, change smoke control setting, override Phase } 2 \text { elevator } \\
\text { control, initiate Phase } 1 \text { recall of protected elevators, shutoff } \\
\text { gas/power/sprinkler, activate emergency voice) }\end{array}$ \\
\hline
\end{tabular}




\section{Message Formats}

The message formats component focuses on how information is packaged to move between different entities. Presently, the primary alert message format standard is the Common Alerting Protocol (CAP) [16] which is under continued development within the Organization for the Advancement of Structured Information Standards (OASIS) Emergency Management Technical Committee. CAP is in use today for public warning systems (e.g., severe weather warnings in the United States and Canada, and chem-bio detection warnings). The NIST Building Information Services and Control System (BISACS) project [17] developed recommended profiles for CAP to support building alert messages. The BISACS architecture envisions CAP alerts from the building propagating from the building SAP, through the emergency dispatch system, and all the way to the responder. Within that alert is a link for the responder to connect back directly to the building to get updated data. An as-yet-undefined set of messages govern how a responder may request data from the building SAP and what responses would be returned.

\section{Message Distribution}

The Message Distribution component addresses the policy for routing messagesdeciding which messages go to which clients and what parts of a message a given client is authorized to access. As shown in Figure 1, there has been an effort to automate alarm reporting from CSA to the PSAP via a message in the National Information Exchange Model (NIEM) format. This effort was supported by the Central Station Alarm Association (CSAA) and the Association of Public-Safety Communications Officials (APCO) and helped to develop the Automated Secure Alarm Protocol, now standard [18].

Many different human users and machine systems may need to access some of the building information before during and after an incident. During an incident, alarm information may be reviewed by a CSA system or user, and a dispatcher will need a presentation of some of the high-level data. The responder will require more information, some of which may come via dispatch, some of which may be saved on a mobile data computer (e.g., building floor plans), and some of which may come directly from the building SAP via a direct connection to get ongoing incident data.

One important protocol for message distribution is the OASIS Emergency Data Exchange Language Distribution Element (EDXL-DE) [19] which covers how alert messages are delivered and received. The EDXL-DE specification has been imported into the NIEM framework by way of an Information Exchange Package Document that specifies how EDXL-DE elements are included within the overall NIEM exchange model [20] As work continues on moving building information to the PSAP and on to the responder, NIEM, buildingSMART Alliance ${ }^{\mathrm{TM}}$, NFPA, NEMA and other standard development organizations may cover distribution requirements for provision of building systems data to incorporate this into a SAP specification. 


\section{Information Technology Infrastructure}

This component addresses the networks for moving messages and the connection between them. Within a building there are multiple networks for building automation and information technology. The building exposes a SAP interface to the external network (Figure 2). The SAP might specify a CAP message that travels across NIEM protocols via the Internet or other network to the central station alarm company, and then on to the NG911 network (NG911 is an internetwork of public safety networks). The PSAP receives a building alert from the NG911 network via a SAP interface and passes the alert through the internal PSAP networks and eventually via radio data network to responders. Responders connect via an external network portal at the building (or back through the public safety network chain) to access ongoing building alerts. The design of a SAP must be developed and agreed upon by building owner and management communities and the owners of the various networks in the chain just discussed for realistic implementation.

\section{Graphical User Interface}

The graphical user interface (GUI) component addresses presentation of data to a user. As noted earlier, the NEMA SB30 standard covers information display formats for fire panels, and this standard is referenced in the NFPA 72 annex. There is ongoing industry user interface research and usability testing that will support updates to the SB30 standard [21, 22, 23].

The specific GUI format and capabilities will depend both on the user's role and current activity. Considering roles, the dispatcher needs high-level information while the IC needs detailed incident information with a user interface that allows drilling down for more detail. Considering activity-based information presentation, previous work has identified two basic information views for the responder: en-route and on-scene. Enroute, the responder needs high-level fire data with a GIS window and building footprint. On-scene, an IC will want real-time fire and smoke data indicated on a floor plan display.

\section{Spatial Representation}

The spatial representation component addresses specifying relationships between things that exist within or occur within a building space and the relative location of those things with respect to the space. The relationship of spaces to other spaces must also be addressed for situational understanding [24]. Enabling visual or auditory presentations on remote devices requires spatial representation. Particularly important standardizations are objects and spaces classification, object location in 3-D space, object location relationship classification, and 2-D plan exchange format. Through standard location and classification support in conjunction with other relevant components, a computer (the responder's) can provide critical emergency response data within the facilities' spatial context on a visual floor plan or through text based description. 
Spatial representation is being addressed by multiple groups, as shown in Table 4. The component work items are presented in rows in Table 4 with cross-references to the various groups or documents that address that work item. Work items include identifying relevant data (summarized in Tables 2 and 3), graphical user interface data import formats, data model for structuring the information relevant to emergency response and associated spatial information, data exchange protocols, types and labels for spaces, and an encoding format for linking real-time data to associated spaces.

Full support for extracting building data for first responder displays requires data classification to be associated with existing building information models, with the main open standard being the Industry Foundation Classes (IFC) [25]. Information categories in Tables 2 and 3 connect building data to the NEMA SB30 display types.

Table 4 Cross-correlation of spatial representation component work items to input sources (projects and specifications) or work groups addressing these work items

\begin{tabular}{|c|c|c|c|c|c|c|c|c|c|c|c|c|}
\hline $\begin{array}{r}\text { Input } \\
\text { providers: } \\
\text { organizations, } \\
\text { projects and existing } \\
\text { recommendations/ } \\
\text { specifications/ } \\
\text { standards } \\
\text { Spatial } \\
\text { Representation } \\
\text { work items }\end{array}$ & 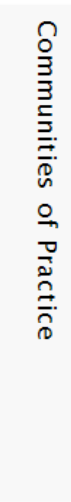 & 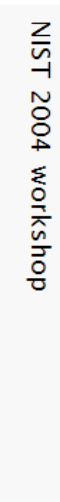 & 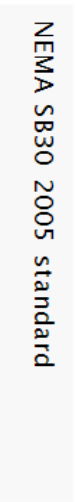 & 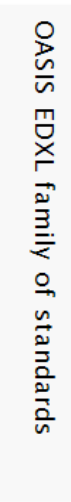 & 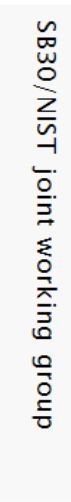 & 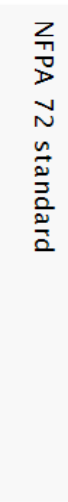 & 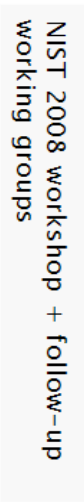 & 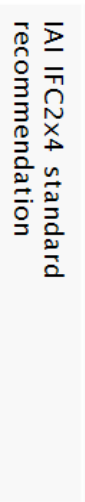 & 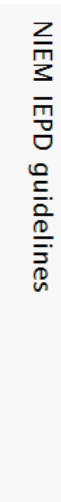 & 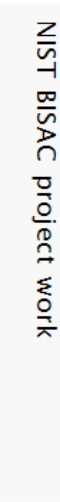 & 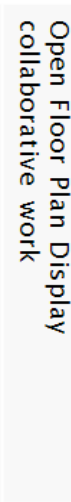 & 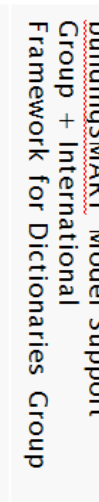 \\
\hline $\begin{array}{l}\text { Required/optional } \\
\text { information for first } \\
\text { responders }\end{array}$ & $x$ & $x$ & $x$ & & $x$ & $x$ & $X$ & $x$ & $X$ & & & \\
\hline $\begin{array}{l}\text { Graphical user interface data } \\
\text { import format }\end{array}$ & $X$ & & $X$ & & $X$ & $X$ & $X$ & $X$ & $X$ & $X$ & $X$ & $X$ \\
\hline $\begin{array}{l}\text { Data model for floor plans } \\
\text { (spatial \& building systems' } \\
\text { information) }\end{array}$ & $X$ & & $X$ & & $x$ & & $X$ & $X$ & $X$ & $X$ & $X$ & $x$ \\
\hline $\begin{array}{l}\text { Floor plan information } \\
\text { exchange }\end{array}$ & $X$ & & & $X$ & $x$ & & $x$ & & $x$ & & $x$ & $X$ \\
\hline Types and labels for spaces & $X$ & & & & & & $X$ & $X$ & $X$ & $X$ & $X$ & $X$ \\
\hline $\begin{array}{l}\text { Relationship Indication for } \\
\text { Real-time Data to } \\
\text { Associated Space Entities }\end{array}$ & $x$ & & & $x$ & $X$ & & $X$ & & & $X$ & $X$ & \\
\hline
\end{tabular}

A more detailed examination of the spatial representation and building information modeling work developments is presented elsewhere. [24-26] 


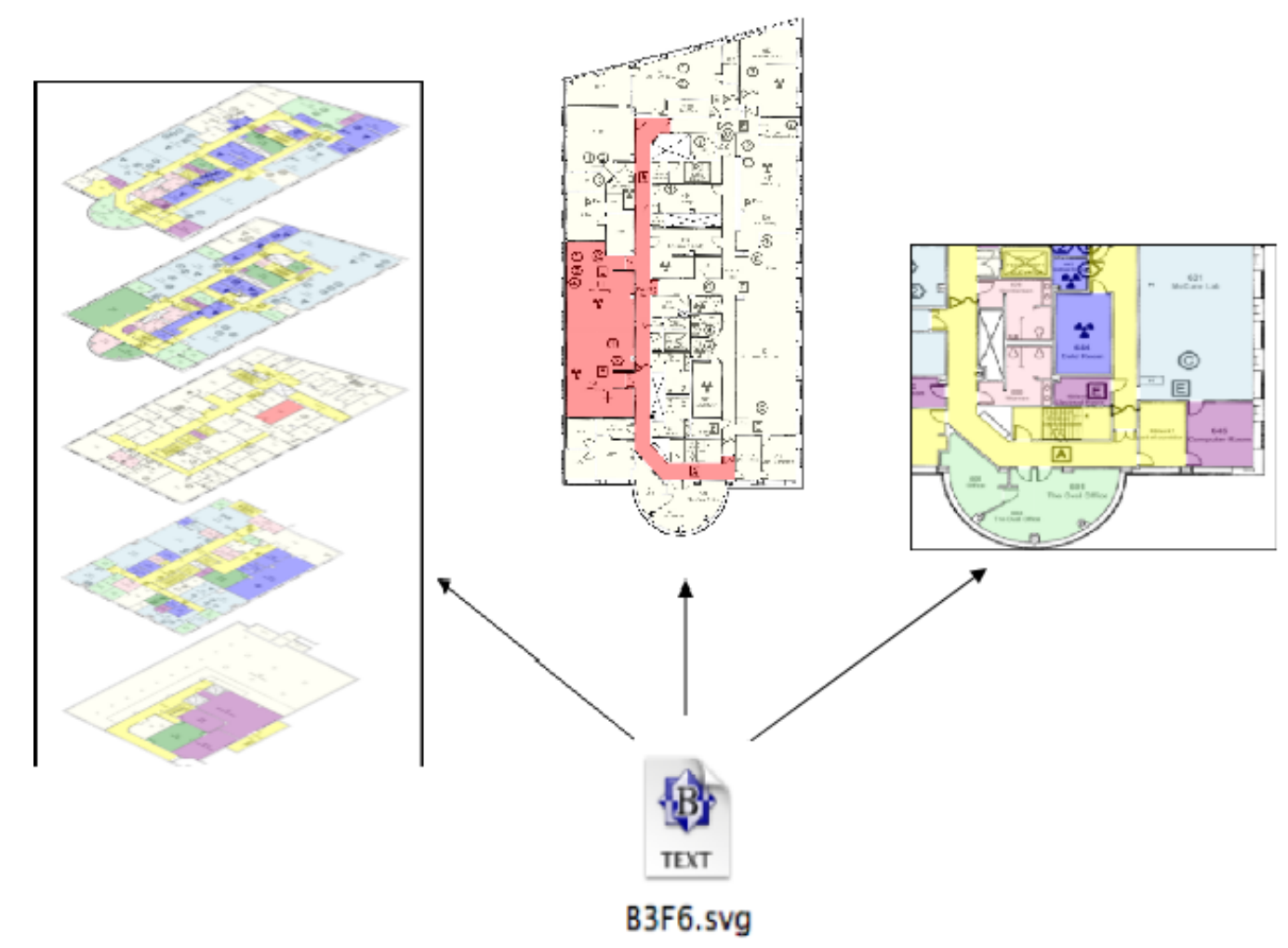

Figure 3 Display graphics are generated from geometry and object properties encoded in SVG, a simple, lightweight file format. The *.svg text file holds the simplified graphical images.

The graphical user interface data import format and floor plan information exchange will be sent over potentially slow or intermittent network connections. A prototype display (depicted in Figure 3) from the Open Floor Plan Display collaborative project [27] utilizes floor plan geometry held in a valid Scalable Vector Graphics (SVG) encoding [28]. The fire response domain specific attributes of the floor plan and information overlay objects are included in the SVG file.

Additional prototyping, usability testing, and interoperability demonstrations are needed.

\section{User Profiles}

The user profiles component focuses on categorization of user roles and standards for process and policy of user access to building information. Following the 2008 workshop, ${ }^{8}$ a work group began to address user role categorization. The user profile work identifies specific roles relative to emergency response and identifies policy as the intersection of user role with incident type and stage of the incident. This work will impact the user role categorizations within the CAP standard. Independently, the IJIS Institute is addressing the security and privacy aspects of user access to data and the buildingSMART Alliance $^{\mathrm{TM}}$ (bSA), the Open Geospatial Consortium, Inc. ${ }^{\circledR}(O G C)$, Open Standards for Real Estate Consortium (OSCRE) and FIATECH are working on process and policy for building information access and sharing. 


\section{Information Security}

Information security remains a significant technical challenge. The NG911 system has the difficult task of allowing open access (anyone can call 911, or in the data sense, send a text message), while simultaneously protecting the system receiving the message from being hacked. It is similar to putting a web server on what has been a public safety private network. From a building information perspective, the challenge is securing the building from unwanted access to building data. Assuming that role-based access control is in place and IT-security standards are in use, there are still other concerns. Given that only authorized users have access to sensitive floor plan data, what are the storage requirements for that data? What procedures are in place to ensure that the data on a mobile computer does not get into the wrong hands? How do we get consistent policies across jurisdictions and for different classes of buildings? These policy issues must be addressed by all the stakeholders as the process moves forward.

\section{Steps Forward}

Enhancing situational awareness and effectiveness of emergency responders will have a significant impact on responder safety and property loss. Maximizing the potential of building data for the responders, however, will require significant effort across a broad spectrum of stakeholders.

Step one is a consensus standard that addresses the content and format of building information presented to responders. Significant progress has been made through NIST and industry collaboration within the NEMA SB30 standardization process.

Step two will be a set of standards that allows an authorized remote client to access realtime building system data via a NEMA SB30 interface (direct connection to the building). In order for any vendor's mobile computer to exchange data with any building system, a standard interface specification is required. Interface standards include: a data model to represent the building information, message syntax, network protocols, security architecture, and mapping of real-time data to a floor plan in the remote unit. With these standards in place, the remote mobile data computer can connect to the building interface and retrieve real-time building incident data and present it on an SB30 display. These first two steps enable access to better information at the fire scene, but do not improve quality of information at the emergency operations center (PSAP).

Step Three addresses automated building alert data transfer from building to PSAP. This effort has begun already with work initiated in APCO, in cooperation with CSAA. The APCO External Alerts Alarm Exchange effort links central station alarm systems via automated messages to the PSAP and was tested in the Richmond, VA area. This effort standardized on the NIEM protocol. At the NIST 2008 workshop, there was discussion of 
the message contents and integration with the NG911 system, with the decision to use the CAP message format encapsulated within NIEM.

Step Four extends the SB30 interface specification to serve as a standard interface on the data networks of Figure 2 (the SAP). Implementing the SAP allows authorized external partners to access building alerts and real-time sensor data. As the work continues, with effective governmental support and with industry coordination, there is reason to believe that the goal of building information for situational awareness is achievable.

\section{Conclusion}

Analyzing and presenting building sensor information to emergency responders has significant potential to improve operational effectiveness and reduce occupational injuries. In order to realize this potential, however, several key technologies and practices must be implemented. Critical factors include building systems data classification and encoding standards, standard network interfaces for accessing information managed by building automation systems, and authorization and authentication architecture agreed upon by public safety network stakeholders.

This paper has reviewed the state-of-the-practice for delivery of building information to emergency responders, presented a framework for information identification and data classification, as well as a roadmap for the remaining challenges. The primary challenges require consensus efforts of multiple stakeholders; however, the benefits to emergency responders, building owners, and building occupants may be substantial.

\section{References}

1. Hall, J., “The Total Cost Of Fire in The United States,” National Fire Protection Association, Quincy, MA, 2008.

2. Jones, W. W.; Holmberg, D. G.; Davis, W. D.; Bushby, S. T.; Reed, K. A. "Workshop to Define Information Needed by Emergency Responders During Building Emergencies.” National Institute of Standards and Technology, Gaithersburg, MD. NISTIR 7193; 34 p. January 2005.

3. NEMA SB 30-2005: Fire Service Annunciator and Interface, National Electrical Manufacturers Association, Rosslyn, Virginia, 2005.

4. “NEMA Introduces New Fire Service Standard”, NEMA News, National Electrical Manufacturers Association, Rosslyn, Virginia, 12 December 2005.

5. NFPA 72: National Fire Alarm Code. 2007 Edition. National Fire Protection Association. Quincy, MA. 2009. 
6. Vettori, R. L.; Lawson, J. R.; Davis, W. D.; Holmberg, D. G.; Bushby, S. T. "HighRise and Large/Complex Incident Communications Workshop. National Institute of Standards and Technology, Gaithersburg, MD. NIST Technical Note 1479; 104 p. February 2007.

7. Davis, W. D.; Holmberg, D. G.; Reneke, P. A.; Brassell, L.; Vettori, R. L. "Demonstration of Real-Time Tactical Decision Aid Displays." National Institute of Standards and Technology, Gaithersburg, MD. NISTIR 7437; 28 p. August 2007.

8. Averill, J., Holmberg, D., Vinh, A., and Davis, W. "Building Information Exchange for First Responders Workshop: Proceedings.” NIST Technical Note 1741, 65 p., August 2009

9. Brooks, R.,Smith, B., Raymond, M., Baclawski, K. “Ontology Applications in Emergency Response,” Ontolog Technical Discussion, January 25, 2007.

10. Jones, E., Webber, D., Brooks, R., Raymond, M., Smith, B.,“'Ontology Applications in Emergency Response (Take 2).” Ontolog Technical Discussion, February 14, 2008.

11. McLean, C. "Role and Implications of Modeling and Simulation for Emergency Preparedness," Exploring the Virtual Organization Landscape: Cyberinfrastrucre Readiness for Emergency Response, Collaborative Expedition Workshop \#77, GSA Intergovernmental Solutions, October 7, 2008.

12. Brooks, R., Coggeshall, D., McLean, C., Radde, G., "Building the Virtual Cyberinfrastructure,” Exploring the Virtual Organization Landscape: Cyberinfrastrucre Readiness for Emergency Response, Collaborative Expedition Workshop \#77, GSA Intergovernmental Solutions, October 7, 2008.

13. Raymond, M., "Adding Building Information Model Resources, Visualization and Information Delivery Systems to the 'Common Operating Picture'," Exploring the Virtual Organization Landscape: Cyberinfrastrucre Readiness for Emergency Response, Collaborative Expedition Workshop \#77, GSA Intergovernmental Solutions, October 7, 2008.

14. Jones, W. W.; Holmberg, D. G.; Davis, W. D.; Bushby, S. T.; Reed, K. A. Workshop to Define Information Needed by Emergency Responders During Building Emergencies. National Institute of Standards and Technology, Gaithersburg, MD. NISTIR 7193; 34 p. January 2005.

15. The OmniClass ${ }^{\mathrm{TM}}$ Construction Classification System, Construction Specification Institute (CSI).

16. Common Alerting Protocol, v. 1.1, OASIS Standard, October 2005.

17. Vinh, A.B., Holmberg, D.G., "Connecting Buildings to Public Safety Networks," NIST Technical Note 1609, June 2009.

18. APCO/CSAA ANS 2.101.1-2008, now rebranded Automated Secure Alarm Protocol (ASAP).

19. Emergency Data Exchange Language - Distribution Element, v 1.0, OASIS Standard, May 2006. 
20. MacPherson, D. L., "Facility Standards and NIEM," National Information Exchange Model (NIEM) Business Architecture Committee, Cannon Design, July 2009.

21. Qu, W., Sun, X., "Interactive Style of 3D Display of Buildings on Touch Screen,” Engineering Psychology and Cognitive Ergonomics, Proceedings of EPCE 2007, Held as Part of HCI International 2007, Springer, 2007. -- ISBN: 978-3-540-73330-0

22. Sun, X., Qu, W., “A Study on the Vertical Navigation of High Rise Buildings,” Engineering Psychology and Cognitive Ergonomics, Proceedings of EPCE 2007, Held as Part of HCI International 2007, Springer, 2007. -- ISBN: 978-3-540-73330-0

23. Zhang, L., Sun, X., Plocher T., “A Research of Speech Signal of Fire Information Display Interface,” Engineering Psychology and Cognitive Ergonomics, Proceedings of EPCE 2007, Held as Part of HCI International 2007, Springer, 2007. -- ISBN: 9783-540-73330-0

24. MacPherson, D., Raymond, M.A., "Ontology Across Building, Emergency, and Energy Standards,” Toward Ontology-based Standards, Ontology Summit 2009, April, 2009.

25. Industry Foundation Classes (IFC) specification, maintained by buildingSmart International, current version available at: http://www.iai-tech.org/.

26. D.L. MacPherson, M.A. Raymond, "Interoperability on a Large Scale," Journal of Building Information Modeling, Spring 2010, p17-18, available at: http://www.wbdg.org/pdfs/jbim_spring10.pdf

27. Open Floor Plan Display (OFPD) collaborative, Project Documentation and Demonstrations, hosted by the SFC MapLab Project, available at http://maplab.org/ofpdocs/.

28. Scalable Vector Graphics (SVG), maintained by the World Wide Web Consortium (W3C), specification available at: http://www.w3.org/Graphics/SVG/. 